\title{
S-18-4 Zinc Status in Liver and Gastrointestinal Diseases
}

\author{
Y.ARAKAWA, ${ }^{2}$ K.SUZUKI, ${ }^{2}$ and S.TAKEUCHI ${ }^{1}$
}

\author{
$1_{\text {Third Department of Internal Medicine }}$ \\ 2 Department of Chemistry, Nihon University School of Medicine, 30-1 Ohyaguchi- \\ Kamimachi, Itabashi-ku Tokyo 173 (Japan)
}

The significances of the biochemical and nutritional roles of trace metals is widely recognized because they are found as constituent components of many metalloproteins and metalloenzymes. In addition, a close correlation between trace metal metabolism and the pathology of hepatic diseases has been recognized based on the facts that such metalloproteins and metalloenzymes are synthesized and react mainly in the liver, and that some trace metals act as cofators against the biosynthesis.

1. Subjects studied

In the present study, the authors have determined serum metals in patients with chronic liver disease by using an inductively coupled plasma emission spectrometry analyzer, and evaluated the pathological significance of them, Furthermore, to clarify the pathogenesis of unbalanced zinc state in patients with chronic liver diseases, the authors have studied the alteration of zinc concentration in the serum and liver from rats with experimentaly $\mathrm{CCl}_{4}$ induced hepatic cirrhosis.

The subjects studied comprised 138 patients with chronic hepatic diseases diagnosed at the 3rd department of Internal Medicine, Nihon University School of Medicine. They consisted of 14 patients with chronic inactive hepatitis, 50 with chronic active hepatitis, 33 with compensatory 1iver cirrhosis, 26 with decompensatory liver cirrhosis and 15 with primary liver cirrhosis (PBC). As controls, 220 adults who exhibited no abnormalities on general biochemical 
tests of their blood were used.

2. Concentrations of serum metals in chronic hepatic diseases.

As shown in Table, the concentration of calcium was low in patients with liver cirrhosis, wheareas it revealed no significant variation in chronic hepatitis or PBC. The concentration of magnesium was low in liver cirrhosis and $\mathrm{PBC}$, but it showed no significant variation in chronic hepatitis. The concentration of phosphorous was low as the disease condition developed from chronic hepatitis to liver cirrhosis, wheareas it was high in PBC. The concentration of iron was high only in chronic active hepatitis. The concentration of copper was high only in liver cirrhosis and PBC. The concentration of zinc was decreased gradually as chronic active hepatitis developed to liver cirrhosis, but it showed no significant changes in chronic inactive hepatitis and PBC. The concentrations of serum calcium and zinc showed marked decreases as compensatory liver cirrhosis developed to decompensatory liver cirrhosis. However, there were no significant differences in the serum concentrations of magnesium, phosphorous, copper and iron between the compensatory and decompensatory stages of liver cirrhosis.

\section{TABLE}

Concentrations of serum metals in patients with chronic liver diseases

\begin{tabular}{cccccc}
\hline \multirow{5}{*}{ Metal } & \multicolumn{5}{c}{ Concentration of serum metal } \\
\cline { 2 - 6 } & $\begin{array}{c}\text { Healthy } \\
\text { controls }\end{array}$ & $\begin{array}{c}\text { Chronic inactive } \\
\text { hepatitis }\end{array}$ & $\begin{array}{l}\text { Chronic active } \\
\text { hepatitis }\end{array}$ & $\begin{array}{c}\text { Liver } \\
\text { cirrhosis }\end{array}$ & $\begin{array}{c}\text { Primary biliary } \\
\text { cirrhosis }\end{array}$ \\
\hline $\mathrm{Ca}$ & $92.2 \pm 4.0$ & $92.9 \pm 8.3$ & $91.4 \pm 6.8$ & $86.5 \pm 8.4^{* * *}$ & $91.7 \pm 3.1$ \\
$\mathrm{Mg}$ & $19.9 \pm 1.4$ & $19.7 \pm 1.2$ & $19.7 \pm 2.2$ & $18.1 \pm 2.2^{* * *}$ & $19.0 \pm 1.4^{* *}$ \\
$\mathrm{P}$ & $124.6 \pm 15.8$ & $114.4 \pm 15.3^{*}$ & $118.2 \pm 20.4^{*}$ & $111.7 \pm 21.5^{* * *}$ & $186.5 \pm 47.0^{* * *}$ \\
$\mathrm{Fe}$ & $1.41 \pm 0.47$ & $1.31 \pm 0.63$ & $1.70 \pm 0.69^{* *}$ & $1.50 \pm 0.83$ & $1.26 \pm 0.48$ \\
$\mathrm{Cu}$ & $0.98 \pm 0.2$ & $1.03 \pm 0.18$ & $1.05 \pm 0.24$ & $1.18 \pm 0.27^{* * *}$ & $1.46 \pm 0.36^{* * * *}$ \\
$\mathrm{Zn}$ & $0.88 \pm 0.1$ & $0.85 \pm 0.13$ & $0.79 \pm 0.2^{* * *}$ & $0.60 \pm 0.18^{* * *}$ & $0.80 \pm 0.18$ \\
\hline \multicolumn{5}{c}{$* \mathrm{P}<0.05, * * \mathrm{P}<0.01, * * * \mathrm{P}<0.001$ as compared with healthy controls. }
\end{tabular}


3. Diagnostic significance of serum $\mathrm{Zn} / \mathrm{Cu}$ ratio

The ratio of the concentration of serum zinc to that of serum copper $(\mathrm{Zn} / \mathrm{Cu}$ ratio) decreased significantly as the morbidity of these chronic hepatic diseases, particularly in the case of liver cirrhosis and $\mathrm{PBC}$. The $\mathrm{Zn} / \mathrm{Cu}$ ratio in the healthy controls ranged from 0.73 to 1.1 , wheareas none of the patients with chronic inactive hepatitis revealed a level of less than 0.5 . Such low levels were observed in 2 (4\%) of 50 patients with chronic active hepatitis, 10 (30\%) of 33 patients with compensatory liver cirrhosis, and 6 (40\%) of 26 patients with decompensatory liver cirrhosis, and $6(40 \%)$ of 15 patients with $\mathrm{PBC}$. Hence, when the $\mathrm{Zn} / \mathrm{Cu}$ ratio is less than 0.5 in chronic hepatic diseases, liver cirrhosis is highly likely.

4. Correlation between concentrations of serum metals and metal-binding proteins.

The concentrations of serum metalloproteins, including prealbumin, albumin, transferrin and retinol-binding protein were significantly decreased in liver cirrhosis, particularly that of the decompensatory type. In this connection, patients with chronic hepatic diseases (chronic hepatitis and liver cirrhosis) were examined for possible correlations between these major metal-binding proteins and the concentrations were observed between calcium $(p<0.05)$ and zinc $(p<0.01)$ and prealbumin, albumin and transferrin, between magnesium and albumin $(p<0.05)$, between phosphrous and retinol-binding protein $(p<0.01)$, and between copper and ceruloplasmin $(p<0.01)$.

The serum concentration of zinc was positively correlated with branched chain amino acids (BCAA) such as valine, leucine and isoleucine (plasma free amino acids), but not with aromatic amino acids (AAA) such as tyrosine and phenylalanine. There was thus a positive correlation between the zinc concentration and $\mathrm{BCAA} / \mathrm{AAA}$ molar ratio (Fischer's ratio). On the other hand, a negative correlation was observed between the zinc concentration and serum III type procollagen N-terminal peptide (PC-III-NP) concentration.

5. Oral zinc tolerance test in patients with chronic hepatic diseases. 
A simple oral zinc tolerance was performed by oral administration of $300 \mathrm{mg}$ sinc sulfate $\left(\mathrm{ZnSO}_{4} \quad 7 \mathrm{H}_{2} \mathrm{O}\right)$ after overnight fasting in patients with chronic hepatic diseases and healthy control subjects. The peak serum concentration at 2 hours after administration and the serum concentration at 3 hours after administration in the patients with liver cirrhosis were lower than those in the healthy controls and patients with chronic hepatitis. Furthermore, the serum zinc curve (AUC) in patients with liver cirrhosis were significantly less than that in patients with chronic hepatitis. These results suggest that the ability of patients with liver cirrhosis to absorb sinc is less than that of healthy controls and patients with chronic hepatitis.

6. Zinc status in experimentally cirrhotic rats treated with $\mathrm{CCl}_{4}$

The authors produced experimental models of liver cirrhosis in rats by $\mathrm{CCl}_{4}$ administration, and determined the concentrations of trace metals in the liver and serum during the process of hepatic fibrosis. To investigate the dynamics of these metals further in vivo, the ${ }^{65}$ Zn uptake by the serum and various organs, including liver, was determined following oral and intravenous administrations of ${ }^{65} \mathrm{Zn}$ to the animals. In addition, $\mathrm{Cu}$ - and $\mathrm{Zn}$-binding substances in the 1iver cytoplasm were analyzed by means of Sephadex G-75 gel chromatography •

The results revealed continuous and significant decreases in zinc and manganese levels in the liver, and significantly lower levels of zinc and higher levels of copper in the serum. Since these metals act as co-enzymes of enzymes involved in cell regeneration and fibrotic changes, the present data indicate that they are probably involved in the process of hepatic fibrosis. The results also suggest the occurrence of decreased zinc absorption in the digestive tract, decreases in the amount of zinc and zinc-binding substances and increases in the amount of copper and copper-binding substances in the liver cytoplasm of rats at the stage of established $\mathrm{CCl}_{4}$-induced liver cirrhosis. 